\title{
Case report: probable transmission of vaccine strain of yellow fever virus to an infant via breast milk
}

\author{
Susan Kuhn MD MSc, Loreto Twele-Montecinos MD, Judy MacDonald MD MCM, Patricia Webster RN, \\ Barbara Law MD
}

\section{- ABSTRACT}

The 17D yellow fever vaccine is a live-virus vaccine that has been in use since the 1940s. The incidence of encephalitis after yellow fever vaccination among young infants is much higher than among children older than nine months of age. Until recently, avoidance of vaccination by breastfeeding women who have received yellow fever vaccine had been based on theoretical grounds only. We report the probable transmission of vaccine strain of yellow fever virus from a mother to her infant through breastfeeding.
$\mathrm{A}$ previously healthy five-week-old male infant presented to hospital with a twoday history of fever and irritability. The day before his admission, he had been noted to have focal seizures on alternating sides. He had poor appetite and had vomited once, but did not develop diarrhea or rash. His fever was preceded by a two-week history of nonspecific rhinorrhea and cough.

A left-sided focal seizure was witnessed in the emergency department. At that time, his temperature was $38^{\circ} \mathrm{C}$, his respiratory rate was 60 breaths/min, his pulse was 164 beats/min and his oxygen saturation was $93 \%$ on room air. He was irritable, with a full anterior fontanelle, nasal congestion, supple neck, and normal tone and reflexes. The remainder of his examination was unremarkable.

The patient had been born vaginally at term. His mother had tested positive on a vaginal swab test for group B streptococcus and had received appropriate intrapartum antibiotics. When the infant was 10 days of age, his mother had received pretravel advice and travel vaccinations. She had been given yellow fever vaccine along with inactivated typhoid vaccine and did not report substantial adverse effects. Three days later, the infant and his mother had departed to Venezuela for one week. They had stayed exclusively in the city of Caracas, and breastfeeding was continued. The baby had showed no insect bites nor had contact with sick people, nor had he been exposed to animals in Canada or abroad. There was no history of herpes infection in family members. He had not received any vaccinations before presentation. Before the onset of his symptoms, his sister (aged five years) and father had upper respiratory tract symptoms. The infant was brought to hospital 20 days after returning to Canada.

Anticonvulsants were administered initially to control seizures, along with empiric ampicillin, cefotaxime and acyclovir at doses appropriate for treating meningitis. Abnormalities on results of a complete blood count included a platelet count of 740 (normal 150-400) $\times 10^{\%} / \mathrm{L}$ and a total leukocyte count of 13.2 (normal 4.0 $11.0) \times 10^{9} / \mathrm{L}$. Serum electrolytes and levels of calcium and magnesium were normal. A computed tomographic scan of the head was normal, and a lumbar puncture was performed. Cerebrospinal fluid parameters were abnormal, with a total leukocyte count of $128.9 \times 10^{6}$ with $30 \%$ neutrophils, $32 \%$ lymphocytes and $36 \%$ monocytes. No red blood cells and no xanthochromia were seen. Glucose was 2.2 (serum 4.3) $\mathrm{mmol} / \mathrm{L}$ and protein was elevated at $1.1 \mathrm{~g} / \mathrm{L}$. Bacterial cultures of blood, urine and cerebrospinal fluid were negative, as was polymerase chain reaction testing of cerebrospinal fluid for herpes simplex virus and enteroviruses. Nucleic acid sequencebased amplification on a nasopharyngeal specimen was also negative for enterovirus.

An electroencephalogram was performed and showed multifocal, potentially epileptogenic discharges. A repeat study 10 days later showed lateral temporal spikes consistent with encephalitis. Magnetic resonance imaging of the head revealed meningeal enhancement in the frontoparietal regions that was consistent with meningoencephalitis. Antibiotics were discontinued after bacterial cultures were reported negative, and the infant was given a 21-day course of acyclovir. His fever resolved after two days, and he
Competing interests: Susan Kuhn received monetary compensation for member expenses from the Committee to Advise on Tropical Medicine and Travel. Barbara Law received monetary compensation for member expenses from the Global Advisory Committee on Vaccine Safety of the World Health Organization. No competing interests declared by Loreto TweleMontecinos, Judy MacDonald and Patricia Webster.

This article has been peer reviewed.

Correspondence to:

Dr. Susan Kuhn, susan.kuhn@albertahealth services.ca

CMAJ 2011. DOI:10.1503 /cmaj.100619 
had no further seizures. He was discharged with no medication after completion of the course of acyclovir and a normal neurologic exam. A follow-up evaluation five months later confirmed normal development and absence of neurologic deficit. The timeline of events is shown in Figure 1 .

A serum sample taken on the patient's admission to hospital was reported to be positive for yellow fever on an IgM capture enzyme-linked immunosorbent assay from the Centers for Disease Control and Prevention. A serum-dilution-plaque-reduction neutralization test for yellow fever was also positive at a titre of 1:5120, and the yellow fever hemagglutination inhibition titre was 1:160. Yellow fever IgG was negative. In addition, a sample of cerebrospinal fluid taken on admission was reported by the Centers for Disease Control and Prevention to be positive for yellow fever antigen by IgM capture enzyme-linked immunosorbent assay, but negative for yellow fever virus by polymerase chain reaction. There were no samples of breast milk available for testing.

Convalescent serum testing four months after admission showed persistence of yellow fever virus $\operatorname{IgM}$, with a drop in the plaque reduction neutralization test titre to $1: 320$, which was suggestive of recent exposure. Serologic testing for western and eastern equine encephalitis, St. Louis encephalitis, Powassan encephalitis and dengue were negative (National Microbiology Laboratory) along with Mayaro, Venezuelan equine encephalitis and West Nile viruses (Centers for Disease Control and Prevention).

\section{Discussion}

The clinical presentation, temporal relationship to maternal vaccination, absence of alternative pathogens and immunologic evidence in both serum and cerebrospinal fluid of the infant were strongly supportive of acute central nervous system infection with vaccine strain of yellow fever. Immunoglobulin $\mathrm{M}$ does not cross the bloodbrain barrier; therefore, its presence in cerebral spinal fluid is indicative of central nervous system infection. The lack of early acute clinical and breast milk specimens made it impossible to retrospectively identify yellow fever virus by molecular methods. Although the infant had visited a country where yellow fever was endemic, he was not in an area that is known to be infected, and there were no known urban outbreaks at the time of his visit. In the absence of any history of vaccination of the child, the only alternative explanation is that he was infected via transmission through breastfeeding by his mother after she was vaccinated. He recovered fully from his illness and had no apparent neurologic deficit as a result.

Figure 1 depicts the timeline of events leading to this infant's presentation based on the known range of incubation periods after infection or vaccination. However, the incubation period for infection via ingestion (i.e., viral infection of and replication in enterocytes before systemic invasion) is unknown. Therefore, the period of incubation after ingestion may be longer than that after enteral exposure with the 17D strain.

Current yellow fever vaccines are live attenuated vaccines produced from the 17D line in embryonated eggs. Serious adverse events

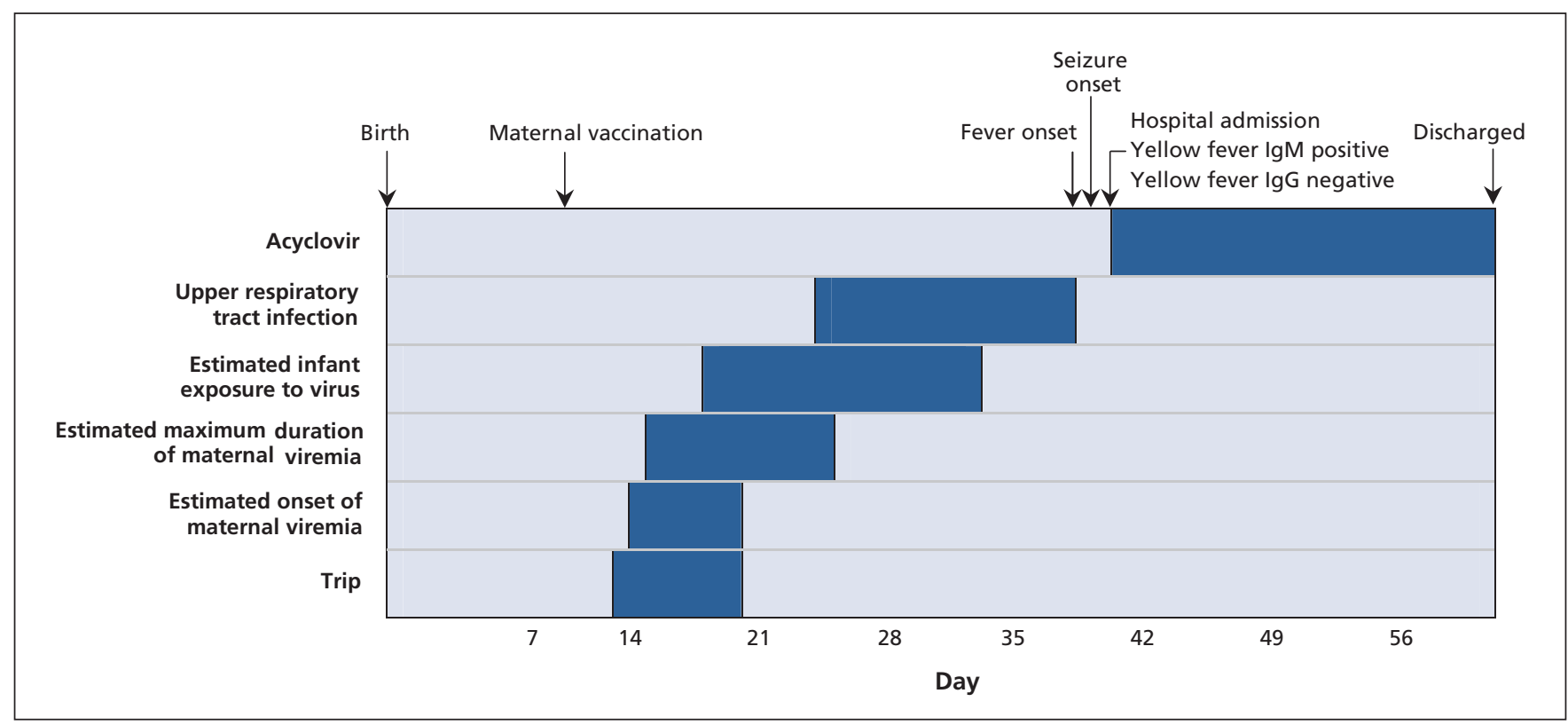

Figure 1: Timeline of events surrounding probable transmission of vaccine strain of yellow fever virus to an infant via breastfeeding. 
include hypersensitivity reactions, viscerotropic disease and neurotropic disease. Vaccine strains are known to retain neurovirulence, and in rare cases cause clinical encephalitis 7-21 days after vaccination, usually with good recovery. This occurrence was first encountered in the 1950s, with $87 \%$ of instances occurring in infants younger than four months of age. ${ }^{1}$ Current guidelines advise avoidance of yellow fever vaccination in infants under age six months and limited use up to age nine months or older. ${ }^{2}$ The reason for the elevated risk is unclear, but it may result from an immature blood-brain barrier, degree of viremia, or immaturity of the immune system. ${ }^{1}$

Previously, recommendations cautioning against use of the vaccine in lactating women had been justified on theoretical grounds only. Canadian recommendations on use of yellow fever vaccine can be found at www.phac-aspc.gc.ca /publicat/cig-gci/p04-yfev-fiej-eng.php. Transmission via blood products after vaccination of the donor was reported earlier this year, showing the risk of contact with body fluid through the parenteral route. ${ }^{3}$ The first probable case of transmission of vaccine strain of yellow fever virus to an infant through breastfeeding was reported only recently from Brazil, although a sample of the breast milk was not available for viral testing. ${ }^{4}$ The infant presented with encephalitis, including increased protein and low glucose but without an elevated leukocyte count, in the cerebrospinal fluid. As in our patient's case, the infant had a good neurologic and developmental outcome.

Experience with other flaviviruses supports the biological plausibility of transmission through breast milk, although the risk of clinical disease is uncertain. Several animal flaviviruses are excreted in milk and cause clinical disease in offspring. ${ }^{5,6,7}$ Transmission through breast milk of West Nile virus in humans has been suspected ${ }^{8}$ but not confirmed.9 Given that excretion of yellow fever virus in breast milk has not been confirmed either, ${ }^{4}$ it is possible that transmission may result from exposure to maternal blood through excoriation of maternal skin and a mucosal break in the mouth of the infant. Regardless, the act of breastfeeding appears to be the most likely mode of transmission of infection to the infant.

This probable instance of yellow fever virus encephalitis further supports the current recommendations $^{2,10}$ for avoidance of yellow fever vaccination in lactating mothers of infants under nine months of age. Although situations may arise that place such a mother at unavoidable and substantial risk for exposure to yellow fever, the risk to the infant from maternal vaccination must be weighed against the risk of wild-type virus infection. Vaccination may still be warranted for lactating women residing in endemic areas during a yellow fever outbreak, for example.

Alternatively, breastfeeding could be avoided during the postvaccination risk period. Onset of viremia can occur between four and ten days after primary vaccination, lasting a mean of two (up to five) days. ${ }^{11}$ Additional data are needed on the duration of excretion of vaccine virus in breast milk to guide recommendations for temporary suspension of breastfeeding after vaccination. However, travellers who are breastfeeding should be strongly encouraged to either adjust their itinerary to reduce or eliminate their risk of exposure (i.e., to avoid the need for vaccination altogether) or postpone their trip until they are no longer breastfeeding and their infant is old enough to be vaccinated with low risk of adverse events.

\section{References}

1. Monath T, Teuwen D, Cetron M. Yellow fever vaccine. In: Plotkin SA, Orenstein WA, Offit PA, editors. Vaccines. 5th ed. Philadelphia (PA): Elsevier; 2008. p. 958-1055.

2. Cetron MS, Marfin AA, Julian KG, et al. Yellow fever vaccine. Recommendations of the Advisory Committee on Immunization Practices. MMWR Recomm Rep. 2002;51:1-11.

3. Centers for Disease Control and Prevention. Transfusion-related transmission of yellow fever vaccine virus: California, 2009. MMWR Morb Mortal Wkly Rep 2010;59:34-7.

4. Centers for Disease Control and Prevention. Transmission of yellow fever vaccine virus through breast-feeding: Brazil, 2009. MMWR Morbid Mortal Wkly Rep 2010;59:130-2.

5. Reid HW, Buxton D, Pow I, et al. Transmission of louping-ill virus in goat milk. Vet Rec 1984;114:163-5.

6. Woodall JP, Roz A. Experimental milk-borne transmission of Powassan virus in the goat. Am J Trop Med Hyg 1977;26:190-2.

7. Shah KV. Experimental infection of lactating monkeys with Kyasanur Forest disease virus. Acta Virol 1965;9:71-5.

8. Centers for Disease Control and Prevention. Possible West Nile virus transmission to an infant through breast-feeding: Michigan, 2002. MMWR Morb Mortal Wkly Rep. 2002;51:877-8.

9. Hinckley AF, O'Leary DR, Hayes EB. Transmission of West Nile virus through human breast milk seems to be rare. Pediatrics 2007;119:e666-71.

10. National Advisory Committee on Immunization. Part 4: Active immunizing agents - yellow fever vaccine. In: Canadian Immunization Guide. Ottawa (ON): Public Health Agency of Canada; 2006.

11. Wheelock EF, Sibley WA. Circulating virus, interferon and antibody after vaccination with the 17-D strain of yellow-fever virus. N Engl J Med 1965;1965:194-8.

Affiliations: From the Departments of Paediatrics (Kuhn, Twele-Montecinos), Medicine (Kuhn) and Community Health Sciences (MacDonald), University of Calgary, Calgary, Alta.; Alberta Health Services, Calgary Zone (MacDonald, Webster); and the Public Health Agency of Canada (Law), Ottawa, Ont.

Contributors: All of the authors contributed to the preparation and revisions of the manuscript and approved the final version submitted for publication.

Acknowledgement: The authors thank the Viral ZoonosesNational Microbiology Laboratory in Winnipeg, Manitoba, for assistance in performing and interpreting the diagnostic tests associated with this case. 